86

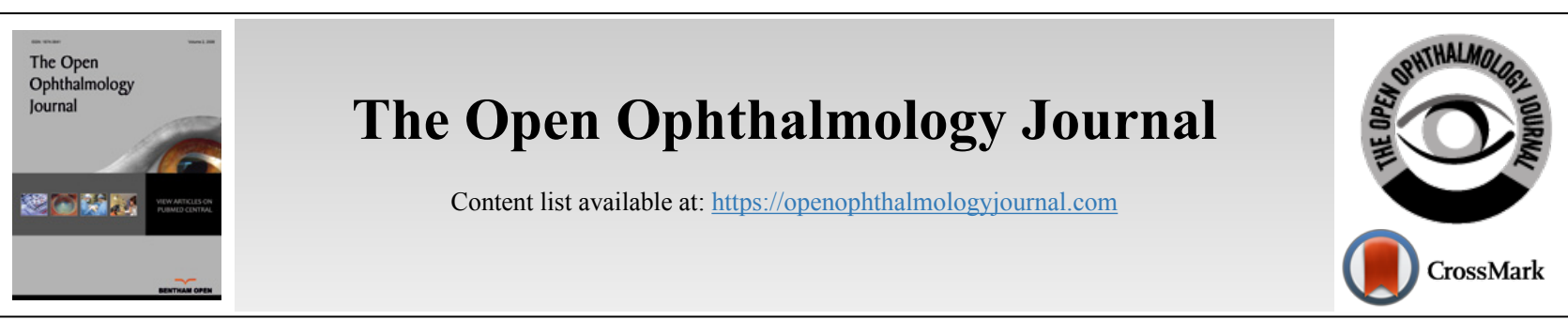

CASE REPORT

\title{
Recurrent Photoreceptor Loss with Spontaneous Recovery as a Presenting Sign of Syphilitic Outer Retinopathy
}

\author{
Jessica G. Shantha', Gregg T. Kokame ${ }^{2,3,4,5,6, *}$ and Lucas Kim ${ }^{7}$ \\ ${ }^{\prime}$ Department of Emory Eye Center, Emory University School of Medicine, Atlanta, GA \\ ${ }^{2}$ Department of Surgery, Division of Ophthalmology, University of Hawaii School of Medicine, 651 IIalo St. Honolulu, HI, \\ ${ }^{3}$ The Retina Center at Pali Momi, HI, USA \\ ${ }^{4}$ Retina Consultants of Hawaii, Honolulu, HI, 96813, USA \\ ${ }_{5}^{5}$ Hawaii Macula and Retina Institute, HI, USA \\ ${ }^{6}$ John A. Burns School of Medicine, University of Hawaii School of Medicine, Honolulu, HI, USA \\ ${ }^{7}$ Mercer University School of Medicine, Macon, GA, USA
}

Abstract: A 64-year-old male presented with sudden onset of decreased vision with a central blind spot and shimmering visual symptoms without significant inflammatory findings, and was noted on optical coherence tomography to have localized loss of photoreceptors, which spontaneously resolved after two weeks. This recurred subsequently three times spaced by months of recovery with normal vision and normal photoreceptors. In the fourth episode, the patient developed an arcuate-shaped area of outer retinitis more typical for acute syphilitic posterior placoid chorioretinitis, and the diagnosis was made serologically. With subsequent treatment for syphilis, there was not any recurrence after the treatment and vision recovered to $20 / 20$ with a normal photoreceptor layer.

Keywords: Syphilis, Outer retinopathy, Acute syphilitic , Posterior placoid chorioretinitis, Ahoroidal thickening, AZOOR.

\begin{tabular}{|l|l|l|r|}
\hline Article History & Received: September 19, 2019 & Revised: November 06, 2019 & Accepted: November 21, 2019
\end{tabular}

\section{INTRODUCTION}

Sudden vision loss associated with localized damage to the photoreceptor layer on Optical Coherence Tomography (OCT) without significant inflammatory signs is an unusual clinical presentation, especially when there is spontaneous resolution with the recovery of normal vision and a normal photoreceptor layer on OCT. Acute Zonal Occult Outer Retinopathy (AZOOR) is an idiopathic disease with acute damage to photoreceptors with minimal funduscopic findings. It is associated with photopsias and is usually found in young females. Acute cone photoreceptor vision loss was recently described as a presenting sign of AZOOR, but the findings persisted and were associated with decreased signal in the photoreceptor layer on OCT [1]. Differential diagnosis includes other acute inflammatory diseases, such as acute multifocal placoid pigment epitheliopathy or unilateral acute idiopathic maculopathy, and infectious diseases, such as tuberculosis and syphilis, sarcoidosis, and intraocular lymphoma.

* Address correspondence to this author at the Department of Surgery Division of Ophthalmology, University of Hawaii School of Medicine, Medical Director, Hawaii Macula and Retina Institute, 98-1079 Moanalua Road, Suite 470, Aiea, Hawaii, 96701, USA; Tel: 1-808-4878928; Fax 1-808-4873699;

E-mail: retinahi@aol.com

\section{CASE REPORT}

A 64-year-old Caucasian male presented with shimmering visual phenomena and a central blind spot in the left eye. Visual acuity was $20 / 25$ in the right eye and 20/50 in the left eye. Slit-lamp examination was normal without iritis, and a dilated fundus exam revealed a focal intraretinal hemorrhage in the macula in the left eye without vitritis. The OCT of the left eye showed diffuse loss of the photoreceptor layer and increased choroidal thickness (Fig. 1A). At two weeks, vision in the left eye spontaneously improved to $20 / 25$ with spontaneous recovery of the photoreceptor layer on OCT (Fig. 1B). The fundus exam of the right eye was normal without hemorrhage or inflammation (Fig. 2A). The OCT of the right eye showed no edema or loss of the photoreceptor layer (Fig. 2B).

Eleven weeks later, he complained of decreased vision in the left eye with vision decreased to $1 / 200$. The funduscopic exam showed retinal whitening and fundus autofluorescence, fluorescein angiography, and OCT angiography findings, which are noted in Fig. (3). OCT displayed subretinal fluid, disruption of the subfoveal cone photoreceptor layer, and choroidal thickening (Fig. 1C). At one month follow up, there was spontaneous recovery of vision to $20 / 30$ with the recovery 
of the photoreceptor layer and resolution of subretinal fluid and outer retinal changes without intervention (Fig. 1D).

Five months later, he complained of central blurring in his left eye with visual acuity of 20/25. Slit-lamp examination and posterior examination were unremarkable with OCT in the left eye showing attenuation of photoreceptor mostly nasally and choroidal thickening (Fig. 1E-1G). The patient's vision spontaneously recovered again with the recovery of the photoreceptor layer.

Fourteen months after the initial presentation, he reported blurry vision in the left eye with visual acuity of 20/40. Dilated fundus examination revealed an arcuate pattern of retinal whitening (Fig. 4). OCT showed disruption of the outer retina with marked loss of the photoreceptor layer. Due to a suspicion of syphilitic posterior placoid chorioretinitis with this more significant presentation, serology was performed for syphilis, which showed a positive FTA-ABS and VDRL (1:64). HIV testing by the infectious disease consultant was negative. He was treated with two weeks of intravenous penicillin $G$ followed by 2.4 million units of intramuscular Bicillin. Two years after the last episode, his vision is $20 / 20$ in both eyes with no recurrences in the left eye and recovery of a normal photoreceptor layer (Fig. 1H). His VDRL decreased from 1:64 to $1: 4$ after treatment.

\section{DISCUSSION}

This case demonstrates the unusual finding of symptomatic vision loss without clinical signs of active inflammation and with loss of the ellipsoid layer on OCT, which rapidly and spontaneously resolved without treatment on three separate episodes with the recovery of good vision and a normal ellipsoid layer. Additional findings included choroidal thickening and decreased flow on OCT angiography isolated to the left eye. Specifically, in the first episode, there is prominent choroidal thickening and an intact External Limiting Membrane (ELM) and disruption of the ellipsoid zone on OCT. While the posterior segment examination did not fit the clinical picture of Typical Acute Syphilitic Posterior Placoid Chorioretinitis (ASPPC), which usually presents as a large yellowish placoid outer retinal lesion, it does suggest an outer retinopathy associated with choroidal inflammation targeting the outer retina. The isolated clinical picture of loss and recovery of the photoreceptor layer on multiple occasions is unique in this case and not previously reported with ocular syphilis. OCT angiography in the second episode showed flow voids in the choriocapillaris, suggesting associated choroidal inflammation. The last episode presented a yellowish-white outer retinal lesion, which was very suggestive of ASPPC, leading to a definitive diagnosis. After the final episode, there was positive serology for syphilis and ASPPC was diagnosed and treated through infectious disease consultation with intravenous penicillin and intramuscular penicillin with no recurrent episodes following appropriate antibiotic treatment over two years.

The imaging, in this case, highlights the acute damage resulting in outer photoreceptor dysfunction, photopsias, and decreased vision. The associated choroidal thickening is suggestive of acute inflammation that may play a role in the pathogenesis of this disease . Evidence in this case for choroidal inflammation includes increased choroidal thickness and punctate hyperreflective dots on OCT and flow voids on OCT angiography at the level of the choriocapillaris. There is one case report by Tsui et al. that supports this notion that flow voids may be seen in ASPPC on OCT angiography [2]. In another case report by Brito et al., they observed choroidal thickening with loss of the ELM and photoreceptors on OCT in unilateral ASPPC [3]. Pichi et al. specifically looked at the OCT findings in ASPPC and found that there was choroidal hyperreflectivity in $14 / 30$ eyes $(46.6 \%)$ again supporting the involvement of the choroid in the pathogenesis [4].

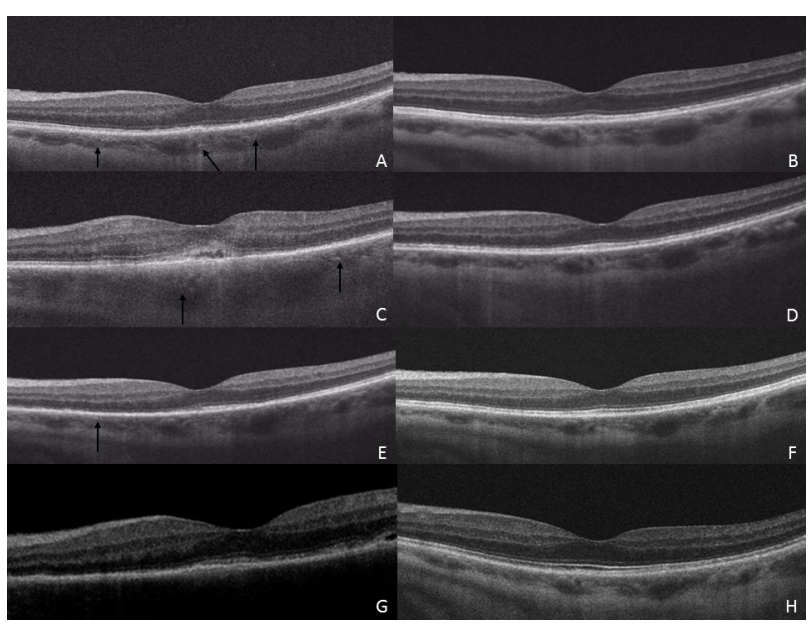

Fig. (1). Optical Coherence Tomography (OCT) of each separate occurrence at presentation and at follow-up after resolution. (A) OCT of initial episode showing diffuse loss of the photoreceptor layer centrally (arrows) with choroidal thickening (B) OCT after vision recovery two weeks later showing recovery of the photoreceptor layer. (C) OCT on presentation after sudden vision loss eleven weeks after first episode. Note the diffuse loss of photoreceptor layer temporally (arrows) and serous detachment under the fovea. The second flare shows choroidal thickening, subretinal fluid, punctate hyperreflective dots (arrows) and outer photoreceptor disruption (C) with spontaneous improvement (D) one month later. The third flare shows choroidal thickening, punctate hyperreflective dots (arrows) and loss of the ellipsoid layer in the nasal macula $(\mathbf{E})$ with spontaneous recovery $(\mathbf{F})$. The fourth flare displays outer photoceptor loss (G) with resolution after systemic treatment $(\mathbf{H})$.

Spontaneous recovery of ASPPC without initial treatment for syphilis has been previously reported $[5,6]$. However, acute loss and then spontaneous recovery of the photoreceptor layer spaced by months of asymptomatic periods with normal vision and photoreceptor recovery are unique presentations of ocular syphilis demonstrated by this case, which mimicked findings seen in acute zonal occult outer retinopathy [1]. The uniqueness of the ocular immune privilege environment may play a role in the ability of the pathogen to elicit an immune response and then resolve spontaneously [4]. Additionally, the pathogen itself, Treponema pallidum, is characterized by phases of latency and its systemic clinical manifestations follow a path defined by current immune status, site of infection, and timing/duration of disease [7]. Similar is the case with the eye that this pathogen is capable of exacerbations and periods of inactivity. 


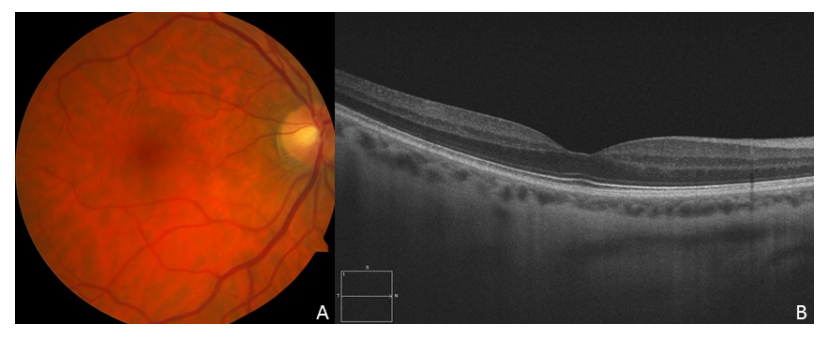

Fig. (2). Color fundus photograph (A) and OCT (B) of the uninvolved right eye. Note the lack of inflammation or edema in the color photograph. Note the normal and intact photoreceptor layer on the OCT.

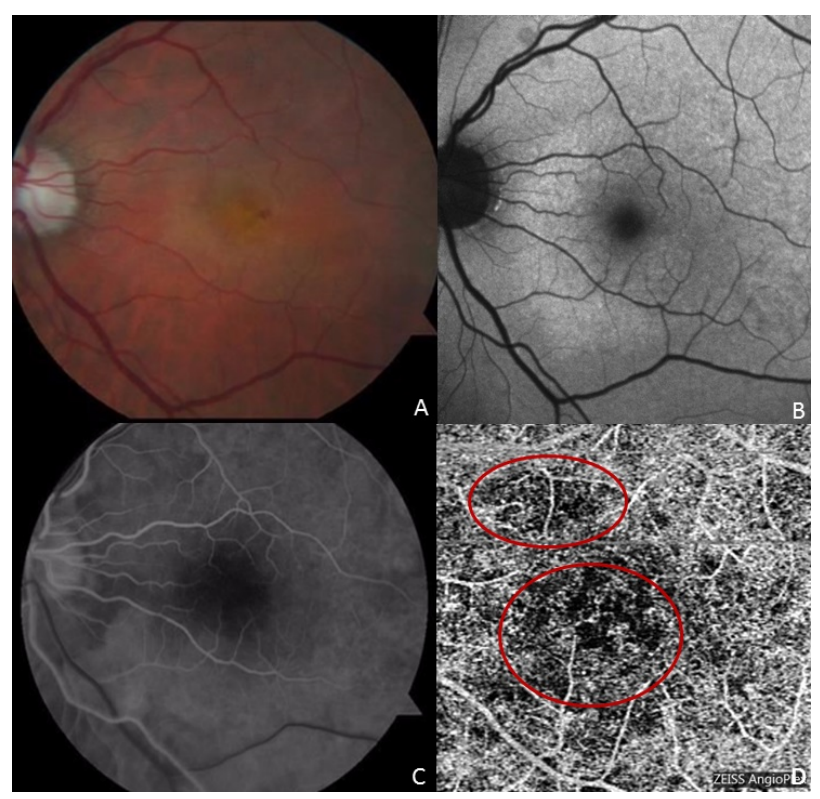

Fig (3). Multimodality imaging from the second flare demonstrates retinal whitening and intraretinal hemorrhage on fundus photo (A) with hyperautofluorescence of the nasal macula on fundus autofluorescence (B) with patchy choroidal filling and delayed perfusion along the inferotemporal vein on fluorescein angiography $(\mathbf{C})$ and flow voids in the left eye on optical coherence tomography angiography (D).

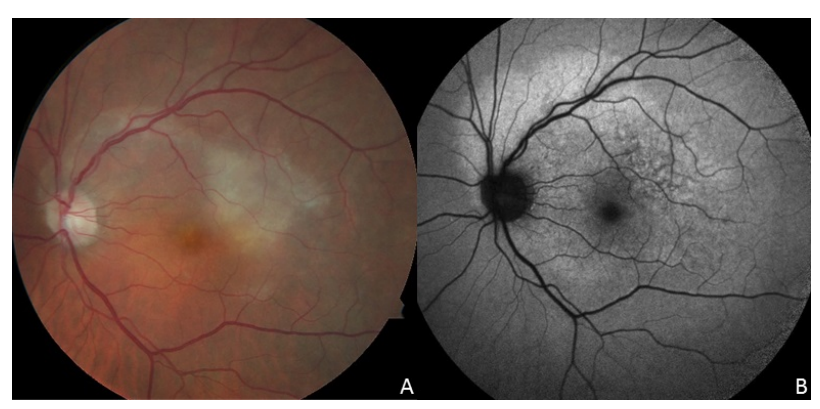

Fig (4). Fundus photo (A) of the fourth flare displays retinal whitening extending from the optic nerve and into the macula with corresponding fundus autofluorescence (B) showing hyperautofluorescence and stippled autofluorescence in the superotemporal macula of the left eye.

\section{CONCLUSION}

This case highlights the unusual presentation of outer retinal damage with loss of the photoreceptor layer on OCT with complete spontaneous recovery for extended periods between episodes. Prior to the diagnosis of syphilis, the presenting findings were considered an unusual variant of acute zonal occult outer retinopathy with central involvement. Understanding the potential presenting clinical spectrum of recurrent and spontaneous resolving outer retinopathy as a possible presenting pattern of syphilis is important, as the treatment of syphilis has now prevented any recurrence with follow-up for over two years while preventing other systemic complications of syphilis.

\section{ETHICS APPROVAL AND CONSENT TO PARTICIPATE}

Not applicable.

\section{HUMAN AND ANIMAL RIGHTS}

Not applicable.

\section{CONSENT FOR PUBLICATION}

Informed consent was taken from all the patients when they were enrolled.

\section{STANDARDS FOR REPORTING}

CARE guidelines and methodology were followed to conduct the study.

\section{FUNDING}

The study reported in this manuscript was supported by Building Interdisciplinary Careers in Women's Health of the National Institutes of Health K12HD085850 (JGS).

\section{CONFLICT OF INTEREST}

Jessica G. Shantha

Consultant: Santen Inc.

Gregg T. Kokame

Research Support: Genentech, Regeneron

Consultant: Genentech, Santen, Regeneron, Bayer, Bausch and Lomb, Zeiss, Ophthotech, Allergan

Speaker: Regeneron, Bayer, Second Sight, Bausch and Lomb, Salutaris

\section{ACKNOWLEDGEMENTS}

The study was presented at the Annual Meeting of the Pan American Association of Ophthalmology, Lima, Peru, August 2017, and at the Annual Meeting of the Midwest Ocular Angiography Conference, Kohala, Hawaii, July 2016.

\section{REFERENCES}

[1] Aleman TS, Sandhu HS, Serrano LW, et al. Acute zonal cone photoreceptor outer segment loss. JAMA Ophthalmol 2017; 135(5): 487-90.

[http://dx.doi.org/10.1001/jamaophthalmol.2017.0451] [PMID: 28384671]

[2] Tsui E, Gal-Or O, Ghadiali Q, Freund KB. Multimodal imaging adds new insights into acute syphilitic posterior placoid chorioretinitis. Retin Cases Brief Rep 2018; 12(Suppl. 1): S3-8.

[http://dx.doi.org/10.1097/ICB.0000000000000645]

[PMID: 29023263] 
[3] Brito P, Penas S, Carneiro A, Palmares J, Reis FF. Spectral-domain optical coherence tomography features of acute syphilitic posterior placoid chorioretinitis: The role of autoimmune response in pathogenesis. Case Rep Ophthalmol 2011; 2(1): 39-44. [http://dx.doi.org/10.1159/000324086] [PMID: 21347191]

[4] Pichi F, Ciardella AP, Cunningham ET Jr, et al. Spectral domain optical coherence tomography findings in patients with acute syphilitic posterior placoid chorioretinopathy. Retina 2014; 34(2): 373-84. [http://dx.doi.org/10.1097/IAE.0b013e3182993f11] [PMID: 23860561]

[5] Baek J, Kim KS, Lee WK. Natural course of untreated acute syphilitic posterior placoid chorioretinitis. Clin Exp Ophthalmol 2016; 44(5):
431-3

[http://dx.doi.org/10.1111/ceo.12679] [PMID: 26566697]

[6] Aranda S, Amer R. Sequential spontaneous resolution of acute syphilitic posterior placoid chorioretinitis. Eur J Ophthalmol 2015; 25(3): 263-5.

[http://dx.doi.org/10.5301/ejo.5000530] [PMID: 25449637]

[7] Carlson JA, Dabiri G, Cribier B, Sell S. The immunopathobiology of syphilis: The manifestations and course of syphilis are determined by the level of delayed-type hypersensitivity. Am J Dermatopathol 2011; 33(5): 433-60. [Review].

[http://dx.doi.org/10.1097/DAD.0b013e3181e8b587]

[PMID: 21694502

\section{C) 2019 Shantha et al.}

This is an open access article distributed under the terms of the Creative Commons Attribution 4.0 International Public License (CC-BY 4.0), a copy of which is available at: https://creativecommons.org/licenses/by/4.0/legalcode. This license permits unrestricted use, distribution, and reproduction in any medium, provided the original author and source are credited. 